\section{Military Technical College Kobry El-Kobbah, Cairo, Egypt}

\author{
$6^{\text {th }}$ International Conference \\ on Electrical Engineering \\ ICEENG 2008
}

\title{
Position fusion of sensors with dissimilar accuracies
}

By

\author{
Ashraf M. Aziz *
}

\section{$\underline{\text { Abstract: }}$}

Position fusion combines locations derived from distributed sensors, monitor an entity, to obtain an accurate estimate of the entity's position. It takes advantages of redundancy and diversity present in the measured data. If the measured data from multiple sensors are correctly combined, then the fused sensor data has better accuracy. This paper addresses the problem of position fusion, based on Bayesian minimum mean square error criterion, using measurement from multiple sensors with dissimilar accuracies. The results show that the performance of the fused measurements may perform worse than the performance of the individual sensor measurements. The best performance of the fused measurements occurs when the sensors have the same accuracies. The performance of the fused measurements is worse than the performance of the best accurate sensor when the sensor accuracies vary widely. In this case, adopting the best accurate sensor is recommenced and position fusion is not recommended.

\section{Keywords:}

Position fusion- Multisensor integration- Data fusion- Tracking using distributed sensors

* Associate Professor, Elec. Eng. Dept., Military Technical College, Cairo, Egypt amaziz64@yahoo.com 


\section{Introduction:}

In recent years, a new discipline called multisensor data fusion has been developed to solve a diverse set of problems having common characteristics. Multisensor data fusion combines data from multiple sensors to perform inferences and achieve performance that may not be possible from a single sensor alone. Data fusion is analogous to the ongoing cognitive process used by humans to integrate data continually from their senses to make inferences about the external world. A human receives sensory data such as sights, sounds, smells, tastes, and touch, which are then combined to reach to a conclusion about the environment. Data fusion spans military and nonmilitary applications. Military applications include ocean surveillance, air-to-air and surface-toair defense, battlefield intelligence, surveillance and target acquisition, and strategic warning and defense. Nonmilitary applications include medical diagnostic, robotics, remote sensing, and automated monitoring of equipments. A summary of these applications bay be found in [1-3].

Ocean surveillance seeks to detect, track, and identify targets. Examples of enemy activities of interest are the launch of a torpedo from a submarine, surface broach of an underwater-launched missile, and communication of a submarine with other vehicles. The ocean surveillance area includes subsurface, surface, and airborne targets in a volume whose dimension may cover a significant portion of the earth. Numerous ships, submarines, and aircraft may be involved in the surveillance process as well as being targets of interest. Observable data include acoustic data, electromagnetic spectrum, nuclear particles, and non nuclear particles. Air-to-air and defense and surface-to-air defense seeks to detect, track and identify aircraft at ranges that permit evasion or deployment of weapons. Systems to identify aircrafts are called IFF (IdentificationFriend-Foe) systems. Primary observable phenomena include electromagnetic radiation (infrared, visible, and radio frequency) utilizing passive and active sensors such as radar, ESM (electronic support measures) receivers, IR cameras, laser radar, and electro-optical sensors (TV). The surveillance volume ranges from hundreds of cubic miles for strategic applications such as the air defense of a country's coastline, to a fewmile radius for defense of a single tactical aircraft. A closely related nonmilitary application is the identification of incoming aircraft to commercial airports. Battlefield intelligence is aimed at the detection and identification of potential ground targets (shooters, or emitters) to infer enemy capabilities, tactics, and strategies. Electromagnetic radiation is a primary observable, including IR emission from enemy engines, RF emissions of communications radios, beacons, radars, visible photographs of an area of interest, and ESM receivers. 
Remote sensing applications include the surveillance of the earth to identify and monitor crops, weather patterns, mineral resources, environmental conditions, and threats (e.g. oil spills, radiation leaks, etc.). Here, the entire spectrum of data may be monitored. Fusion systems are also being developed to monitor and control complex equipment and manufacturing processes. Certain systems, such as nuclear power plants and modern aircraft, require monitoring beyond the ability of a human operator. Automated monitoring is required to ensure that the system continues to operate properly. Data from multiple sensors is monitored to assess the health of a system. A number of data fusion systems have also been developed for automatic fault diagnosis of complex equipment. Inferences range from simple monitoring of equipment's function (e.g., the output data - temperature, speed, pressure - within acceptable ranges) to complex inferences (e.g., are there indications of an impending meltdown?) involving many possible observations and indicators. Other examples of data fusion are the techniques employed for medical diagnosis. At a basic level, to diagnose common illnesses, a physician may use touch (feeling a patient's skin, checking the motion of a joint), sight (observing a patient's complexion, evidence of ear obstructions, etc.) and sound (listening to breathing), as well as a patient's self reported symptoms. More complex problems may involve obtaining multiple sensor data (e.g. x-ray images, nuclear magnetic resonance, chemical and biological tests, ultrasound) and other data to determine the condition of a patient. A final example of fusion applications is the integration of multiple sensor data for robotic applications. Industrial robots use pattern recognition and inference techniques to recognize three-dimensional objects. All the previous examples certainly do not exhaust the range of problems for which data fusion techniques are applicable.

This paper addresses the position fusion which is one of the important main functions of data fusion processing. The remainder of this paper is organized as follows. A brief review of data fusion model and the data fusion processing are addressed in Section II. Position fusion is presented in Section III. Performance evaluation of position fusion in distributed multisensor systems and numerical results based on Monte Carlo simulations are reported in Section IV. Section V contains conclusion.

\section{Processing in a Data Fusion Model:}

The input to a data fusion system consists of sensor data, commands, and a priori data, and the output from a data fusion system represents the combined or fused data. These output data represents specific and accurate estimates of the location and identity of a target, entity, activity, or situation. Often, a hierarchy of inferences may be sought as shown in Fig. $1[1,3]$. At the most basic level, a sensor suite and fusion system seeks to detect energy and to determine the existence of an entity or situation. 
Here the term entity will be used to indicate a physical phenomenon, platform or vehicle, enemy unit, target, presence and identity of an equipment fault, source of illness, or other situation for which the fusion system was intended to observe. Clearly the entity referenced depends on the specific application. Given the suspected existence of an entity, the next level of inference to be developed is the position and velocity of the entity. Ideally, a complete positional state vector is sought having six dimensions: three components specifying position ( $\mathrm{x}, \mathrm{y}, \mathrm{z}$ ), and three components specifying velocity. The concept of state will be defined in the next section. Briefly, the state vector is comprised of those parameters that, if known accurately, would allow the prediction of the future of the system.

Closely related to position is the identity of the entity. Identity might consist of a general classification of which the observed aircraft is a member; a class of fighter

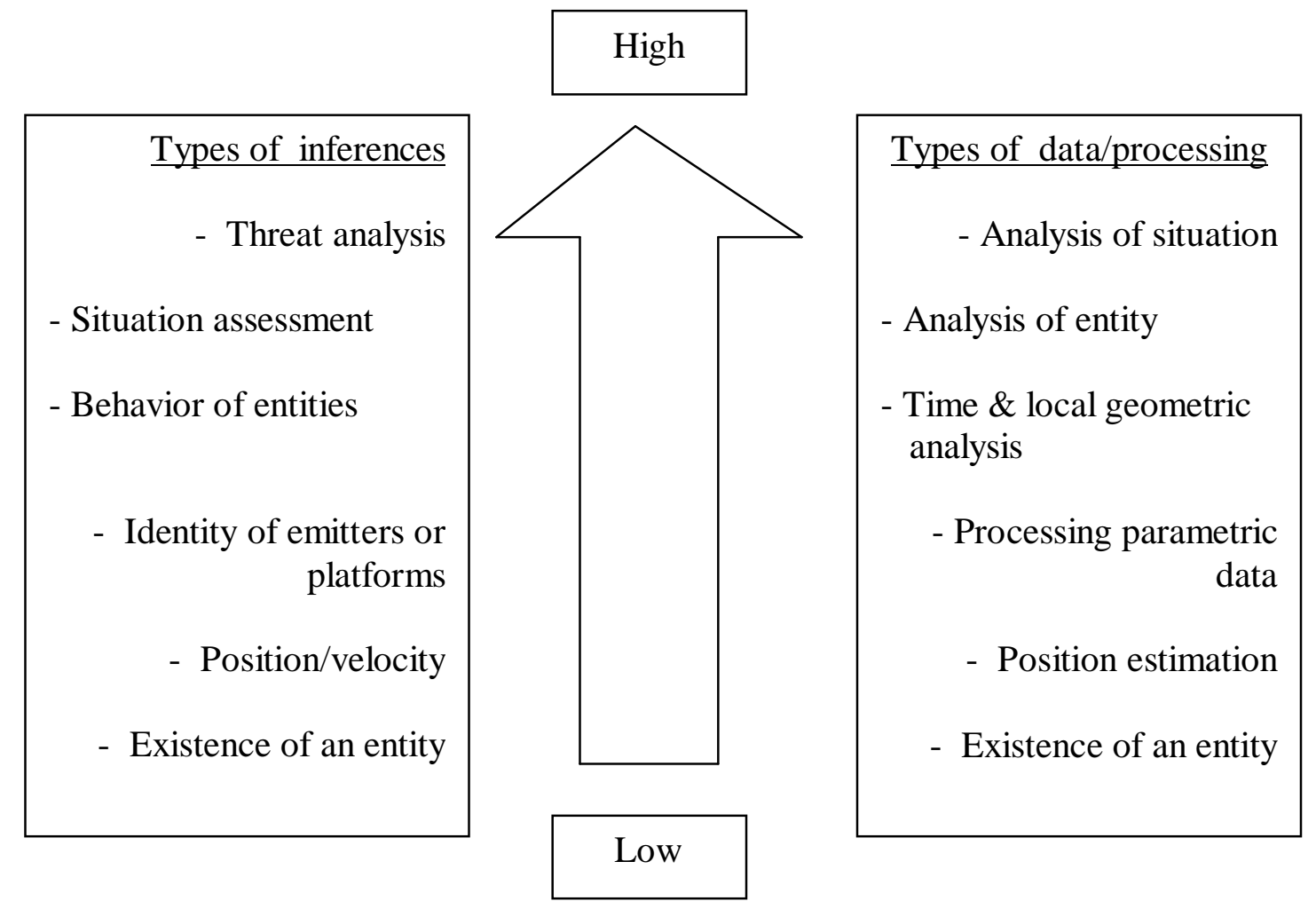

aircraft; a specific classification within a general class (e.g., the observed aircraft is an F-16); or a specific identity (e.g., the observed aircraft is an F-16 having tail number 12345SC). Inference of an entity's involves transformations from parametric data to identity declarations utilizing pattern recognition methods. Higher level of inference involve the behavior of entities, intent, situation assessment, and (for military systems) assessment of threat. Progression up the inference hierarchy pictured in Fig. 1 involves utilization of techniques ranging from signal processing algorithms and statistical estimation methods for combining parametric data, to heuristic methods for threat analysis [4-6]. 
Figure 2 illustrates a functional model for the data fusion process. This functional model is based on a presentation by Llinas and Hall [4]. It incorporates three basic levels of processing: level 1,2 , and 3 . The input data to the model may be sensor data, a priori data, and human inferences. Level 1 processing consists of positional fusion, which combines locations derived from all sensor data to obtain the most accurate estimate of an entity's position and velocity, and identity fusion, which combines data related to the identity of entities (e.g., classification of entities into classes and the identity of an enemy aircraft, ship, or emitter). The term entity refers to a target, an emitter or platform.

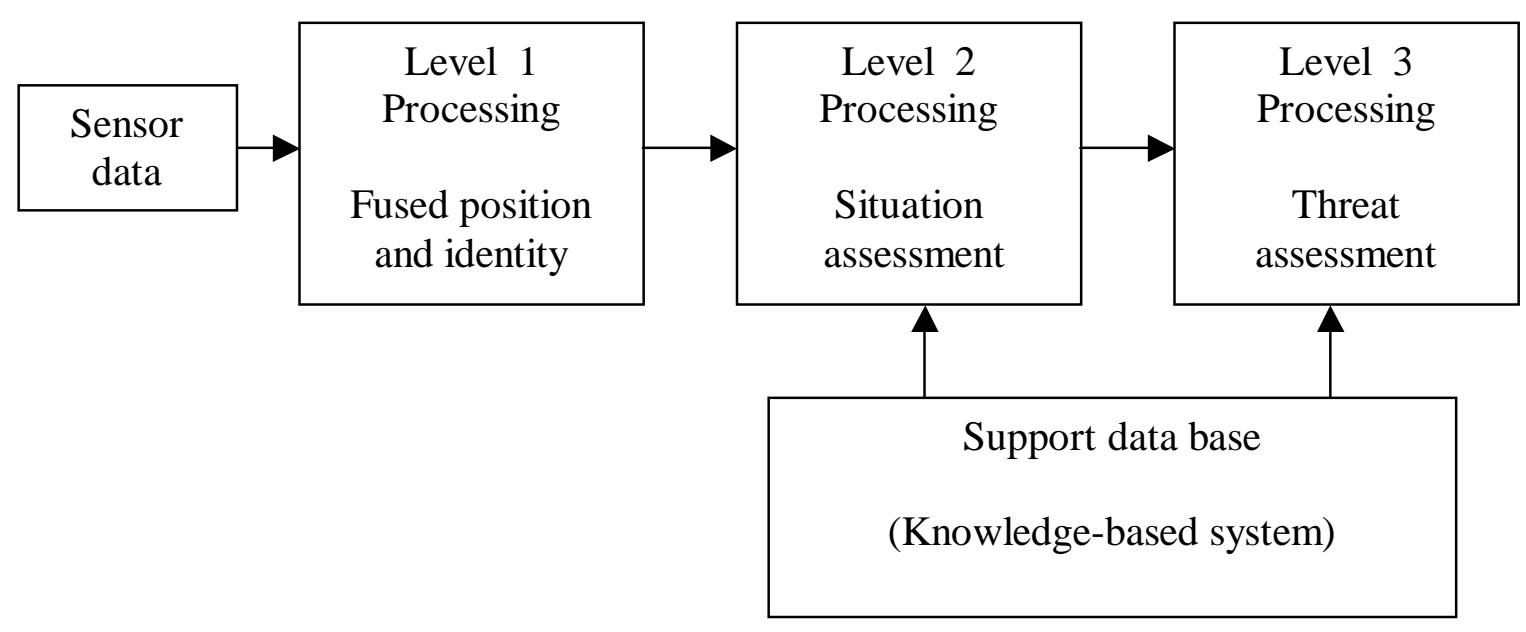

Fig.2 Data fusion model

The positional fusion is divided into two subgroups: parametric association and estimation techniques [7-14]. Parametric association correlates observations from multiple sensors to individual entities (tracks). That is, multiple targets are observed by multiple sensors, and the association between observations and targets is not known a priori [15-18]. Parametric association is important because an incorrect data association would affect the performance of the target tracking. Given the association of each observation to each target, estimation techniques are then used to combine the data to obtain better estimate of the state vector attributes (such as position and velocity). Level 2 processing is aimed at situation assessment, a process by which a description of the relationships among all entities is developed. In this level, the outputs from level 1 processing are analyzed and examined to bring out the essential features of the distributed combat units and weapon systems. Level 3 processing is used for threat assessment. Its purpose is to determine the meaning of the fused data, such as an estimate of enemy lethality, expected courses of action, unit compositions and deployment, and an estimate of threat. This level usually employs heuristic techniques similar to those for situation assessment in addition to utilizing the knowledge-based systems or expert systems (support data base) [19-21]. 
As shown in Fig. 3, level 1 processing performs four main functions: (1) data alignment, (2) data association, (3) tracking, and (4) identification [1, 2-5]. The data alignment function transforms sensor data into a common set of coordinates and units. Converting the data received from each sensor to a common coordinate system is necessary to fuse target information from dissimilar sensors. Dissimilar sensors mean sensors with different accuracies (or resolutions). The data association function correlates the sensors data to entities, and the tracking function combines positional data to yield an estimate of the target state vector. The two main techniques used for estimation are batch estimation techniques and sequential estimation techniques [22-24]. The identification function combines information to determine the identity of entities (classification of targets). We focus on the positional fusion in the remainder of this paper.

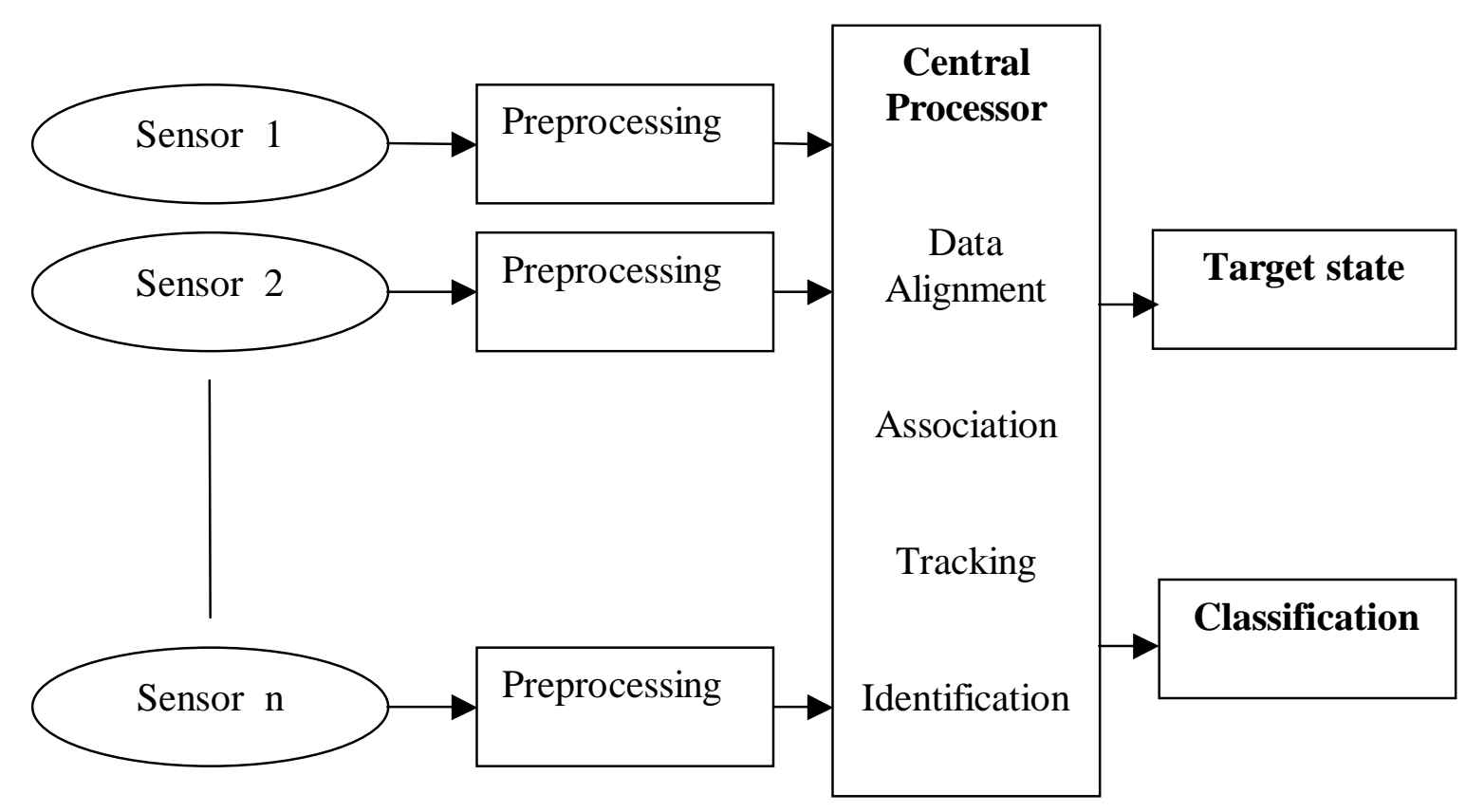

Fig.3 Level 1 processing

\section{Position Fusion Using Multiple Sensors:}

Willner et al. [25] introduced the Kalman filter algorithms for multisensor multitarget systems. They use a test statistic to determine whether or not two tracks are the same and solve the problem of track fusion assuming independent estimation errors. There are two different implementations of the fusion algorithms: parallel and sequential $[25,26]$. In a parallel fuser, the estimates $\hat{\mathbf{x}}_{i}$ with the corresponding covariance $\mathbf{P}_{i}$ are applied simultaneously to the fusion center. 
The fused estimate, which minimizes the expected mean square error, and the corresponding covariance are given by:

$$
\begin{aligned}
& \mathbf{X}_{f}=\mathbf{P} \sum_{i=1}^{n} \mathbf{P}_{i}^{-1} \hat{\mathbf{x}}_{i}, \ldots \ldots \\
& \mathbf{P}^{-1}=\sum_{i=1}^{n} \mathbf{P}_{i}^{-1}
\end{aligned}
$$

where $n$ is the total number of the tracks considered in the fusion. In a sequential fuser, the estimates are fused sequentially. The fused estimates of any two tracks $\hat{\mathbf{x}}_{i}$ and $\hat{\mathbf{x}}_{j}$ are given by [24-26]:

$$
\begin{aligned}
& \mathbf{X}_{f}=\mathbf{P}_{j}\left(\mathbf{P}_{i}+\mathbf{P}_{j}\right)^{-1} \hat{\mathbf{x}}_{i}+\mathbf{P}_{i}\left(\mathbf{P}_{i}+\mathbf{P}_{j}\right)^{-1} \hat{\mathbf{x}}_{j}, \ldots \\
& \mathbf{P}=\mathbf{P}_{i}\left(\mathbf{P}_{i}+\mathbf{P}_{j}\right)^{-1} \mathbf{P}_{j} \ldots \ldots
\end{aligned}
$$

The sequential and the parallel fusers are equivalent in terms of the accuracy of the fused estimate. However the sequential fuser is faster than the parallel fuser since it requires ( $n-1)$ inversions of $M \times M$ covariance matrix as opposed to (2n-1) inversions of the same matrix in the parallel fuser.

Bar-Shalom $[15,26]$ retreated the problems of track-to-track association and track fusion under the assumption that the estimation errors of different sensors are correlated. Bar-Shalom mentioned that the measurement noises of two different sensors can be assumed dependent but is not sufficient to yield the independence of their estimation errors. This is because the same process noise in the dynamic model makes the two estimation errors correlated [27-29]. By assuming that the difference between two estimates from different systems (sensors) $i$ and $j$ is

$$
\mathbf{d}_{i j}=\hat{\mathbf{x}}_{i}-\hat{\mathbf{x}}_{j}, \ldots \ldots
$$

the covariance of the difference of the two estimates is

$$
E\left\{\mathbf{d}_{i j} \mathbf{d}_{i j}^{\prime}\right\}=E\left\{\left(\hat{\mathbf{x}}_{i}-\hat{\mathbf{x}}_{j}\right)\left(\hat{\mathbf{x}}_{i}-\hat{\mathbf{x}}_{j}\right)^{\prime}\right\}
$$

The covariance of the difference can be rewritten as

$$
\begin{aligned}
E\left\{\mathbf{d}_{i j} \mathbf{d}_{i j}^{\prime}\right\} & =E\left\{\left(\hat{\mathbf{x}}_{i}-\mathbf{x}-\left(\hat{\mathbf{x}}_{j}-\mathbf{x}\right)\right)\left(\hat{\mathbf{x}}_{i}-\mathbf{x}-\left(\hat{\mathbf{x}}_{j}-\mathbf{x}\right)\right)^{\prime}\right\} \\
& =\mathbf{P}_{i}+\mathbf{P}_{j}-\mathbf{P}_{i j}-\mathbf{P}_{j i},
\end{aligned}
$$

where $\mathbf{P}_{i j}$ represents the cross-correlation between the two estimates and is given by $\mathbf{P}_{i j}=E\left\{\left(\hat{\mathbf{x}}_{i}-\mathbf{x}\right)\left(\hat{\mathbf{x}}_{j}-\mathbf{x}\right)^{\prime}\right\}=E\left\{\tilde{\mathbf{x}}_{i} \tilde{\mathbf{x}}_{j}\right\}=\mathbf{P}_{j i}$ 
In this case, the results of the fused estimate and the corresponding covariance which minimize the mean square error (MSE) will be

$$
\begin{aligned}
& \mathbf{X}_{f}=\hat{\mathbf{x}}_{i}+\left(\mathbf{P}_{i}-\mathbf{P}_{i j}\right)\left(\mathbf{P}_{i}+\mathbf{P}_{j}-\mathbf{P}_{i j}-\mathbf{P}_{i j}^{\prime}\right)^{-1}\left(\hat{\mathbf{x}}_{j}-\hat{\mathbf{x}}_{i}\right), \ldots \ldots \ldots \\
& \mathbf{P}=\mathbf{P}_{i}-\left(\mathbf{P}_{i}-\mathbf{P}_{i j}\right)\left(\mathbf{P}_{i}+\mathbf{P}_{j}-\mathbf{P}_{i j}-\mathbf{P}_{i j}^{\prime}\right)\left(\mathbf{P}_{i}-\mathbf{P}_{i j}^{\prime}\right), \ldots \ldots
\end{aligned}
$$

where $\mathbf{P}_{i j}$ is determined from the following recursive equation

$$
\mathbf{P}_{i j}=(I-\mathbf{K} \mathbf{H})\left(\mathbf{F} \mathbf{P}_{i j} \mathbf{F}^{\prime}+\mathbf{Q}\right)(I-\mathbf{K} \mathbf{H})^{\prime},
$$

where $\mathbf{K}$ is the Kalman filter gain, $\mathbf{F}$ is the state transition matrix, $\mathbf{Q}$ is the plant noise covariance matrix, and $\mathbf{H}$ is the measurement matrix.

\section{Monte Carlo Simulations:}

By assuming that the targets are moving in straight lines with constant velocities, the targets motion model at scan $k$ will be [30]

$\mathbf{x}(k+1)=\mathbf{F} \mathbf{x}(k)+\mathbf{g}(k)$,

where $\mathbf{g}(k)$ is the plant noise and $\mathbf{F}$ is the state transition matrix given by

$$
\mathbf{F}=\left(\begin{array}{cccc}
1 & T_{s} & 0 & 0 \\
0 & 1 & 0 & 0 \\
0 & 0 & 1 & T_{s} \\
0 & 0 & 0 & 1
\end{array}\right)
$$

and $T_{s}$ is the sampling interval.

The state vector $\mathbf{x}(k)$ contains the $\mathrm{x}$ - and $\mathrm{y}$ - target positions and velocities, i.e.

$$
\mathbf{x}(k)=\left(\begin{array}{c}
x(k) \\
v_{x}(k) \\
y(k) \\
v_{y}(k)
\end{array}\right) \ldots
$$

The measurements are the $\mathrm{x}$ - and $\mathrm{y}$ - target positions, i.e.

$$
\mathbf{z}(K)=\mathbf{H}(K) \mathbf{x}(K)+\mathbf{w}(K),
$$

where $\mathbf{H}$ is the measurement matrix given by 


$$
\mathbf{H}=\left(\begin{array}{llll}
1 & 0 & 0 & 0 \\
0 & 0 & 1 & 0
\end{array}\right) \ldots \ldots \ldots \ldots \ldots \ldots \ldots(16)
$$

Measurements are affected by noise which is modeled as Gaussian, zero mean, with a certain standard deviation. The noise sequence $\mathbf{w}(K)$ has a covariance matrix

$$
\mathbf{C}_{K}=\left(\begin{array}{cc}
\sigma_{i j}^{2} & 0 \\
0 & \sigma_{i j}^{2}
\end{array}\right), \ldots \ldots \ldots \ldots \ldots . . .(17)
$$

where $\sigma_{i j}^{2}$ represents the variance of the measurement error due to observing target $i$ by sensor $j$.

The performance is evaluated based on 500-run Monte Carlo simulations. The measurement noise is assumed to be Gaussian. The measurement error is defined as $e=\sqrt{e_{x}^{2}+e_{y}^{2}}=\sqrt{\left(\mathbf{x}_{\text {true }}-\hat{\mathbf{x}}\right)^{2}+\left(\mathbf{y}_{\text {true }}-\hat{\mathbf{y}}\right)^{2}}$

where $\mathbf{x}_{\text {true }}$ and $\mathbf{y}_{\text {true }}$ are the true (actual) target trajectories while $\hat{\mathbf{x}}$ and $\hat{\mathbf{y}}$ are the estimated target trajectories. The performances of the superior track and the fused track are compared in terms of the mean measurements errors for different values of sensor accuracies (noise variances) assuming independent estimation errors. The sensor accuracies are defined in terms of the noise standard deviation for each sensor assuming common standard deviation in both $\mathrm{x}$ - and y-positions, i.e.

$\mathrm{SD}_{x n}=\mathrm{SD}_{y n}=\mathrm{SD}_{n}, n=1,2$,

We consider the case of a two crossing targets with two attributes; $\mathrm{x}$ - and y-positions. The two targets are observed and detected by a number of sensors $n$. The actual targets trajectories are shown in Fig. 4. The actual and measured trajectories are shown in Fig. 5. The actual and Kalman filter trajectories are shown in Fig. 6. The mean measurements errors for both targets are shown in Fig. 7 assuming a single sensor. The mean measurements errors for target 1 for different values of identical sensors $n$ are shown in Fig. 8 assuming that the common noise standard deviation is $50 \mathrm{~m}$. Fig. 9 shows the mean measurements errors for target 1 for different values of identical sensors $n$ assuming that the common noise standard deviation is $250 \mathrm{~m}$. The mean measurements errors for target 1 in case of non identical sensors are shown in Fig. 10 13. Fig. 10 compares the mean measurements errors of the superior (better quality sensor estimate) and the fused tracks in case of comparable sensors accuracies. Fig. 11 shows the same plot in case of small difference in sensors accuracies. Fig. 12 compares the mean measurements errors of the superior and the fused tracks in case of large difference in sensors accuracies. Fig. 13 shows the same plot in case of very large difference in sensors accuracies. 
As shown in Figures 8 and 9, the performance of the fused track is better than the performance of a single sensor system in case of identical sensors (sensors with similar accuracies). From Figures 8 and 9, the performance increases as the number of sensors increases. It means that the mean measurements errors decrease as the number of sensors increases. In case of non identical sensors, the results show that the performance of the fused track may be worse than the performance of the superior track. From Figures 10 and 11 it is clear that the performance of the fused track is better than the performance of the superior track when the sensors have similar or comparable accuracies. Fig. 12 and Fig.13 show that the performance of the fused track is worse than the performance of the superior track when the sensor accuracies vary widely. The results show that the performance of the fused estimate is marginally better than that of the better quality sensor estimate when the sensors are dissimilar (with different sensor accuracies). The best performance of the fused estimate occurs when the distributed sensors are similar (having the same accuracies). The performance of the fused track is worse than the performance of the better quality estimate when the sensors accuracies vary widely. In this case, it is recommended to adopt the estimate of the better quality sensor and the fusion of sensor estimates is not recommended.

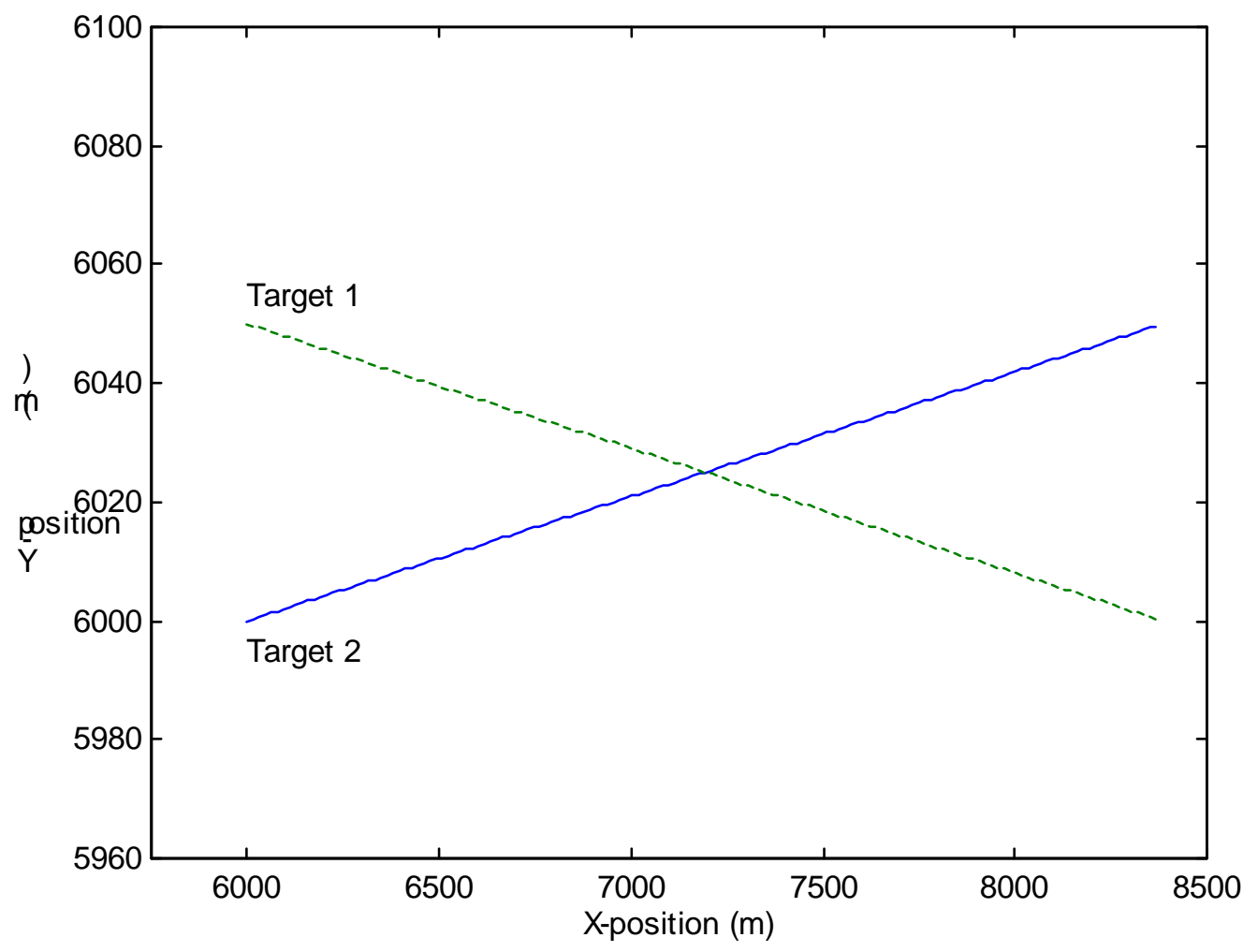

Fig. 4 Actual targets trajectories 


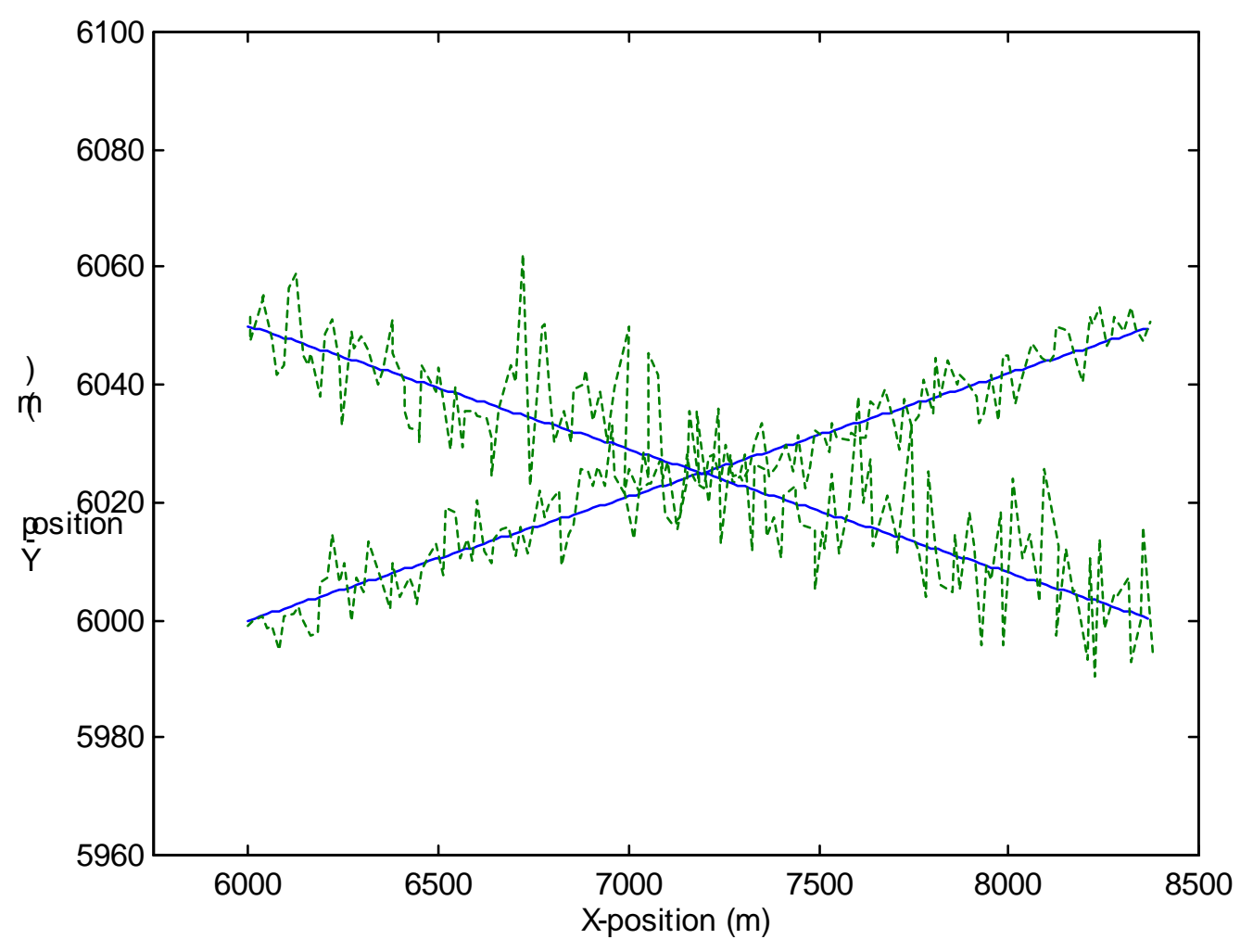

Fig. 5 Actual and measured targets trajectories

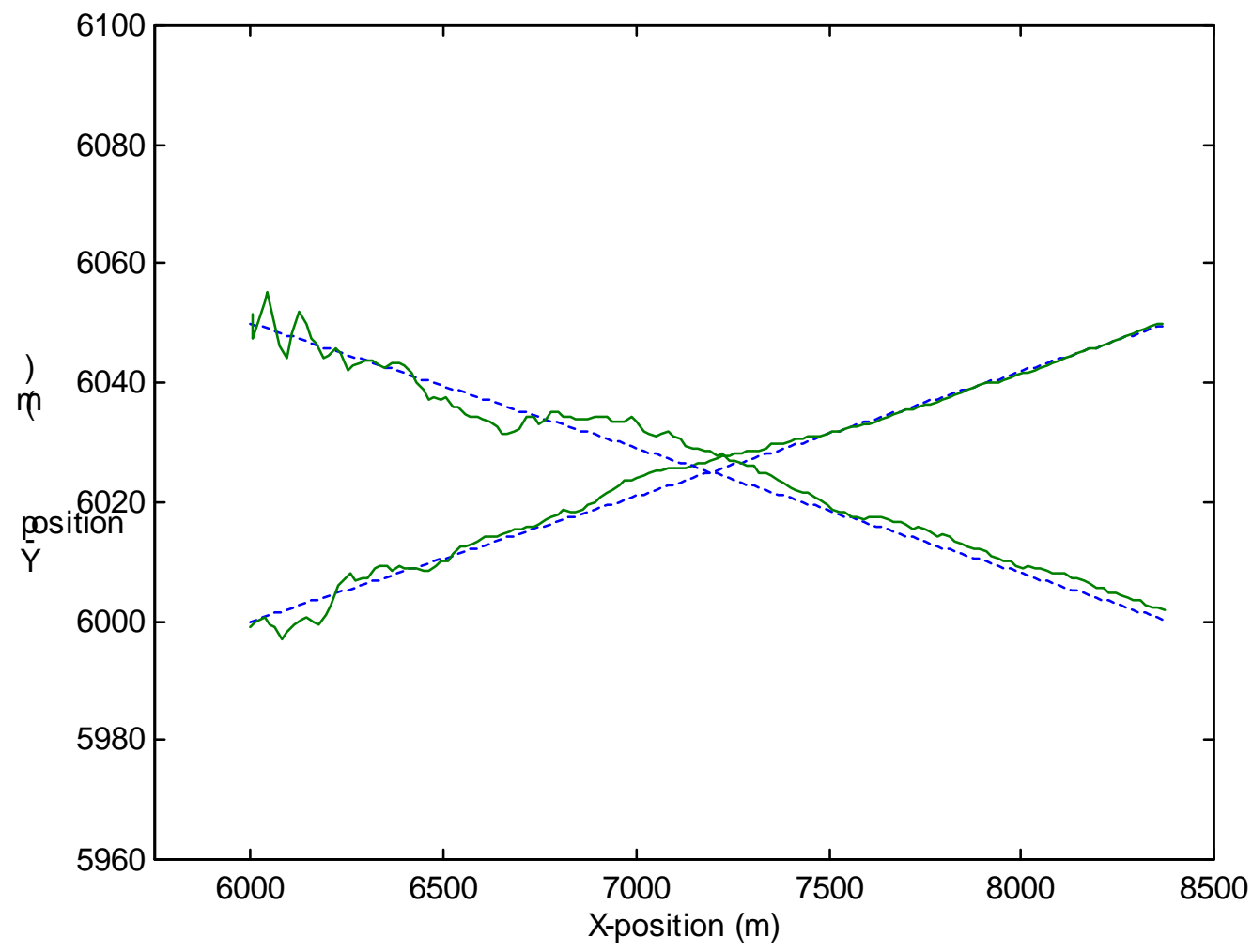

Fig. 6 Actual and Kalman Filter trajectories 


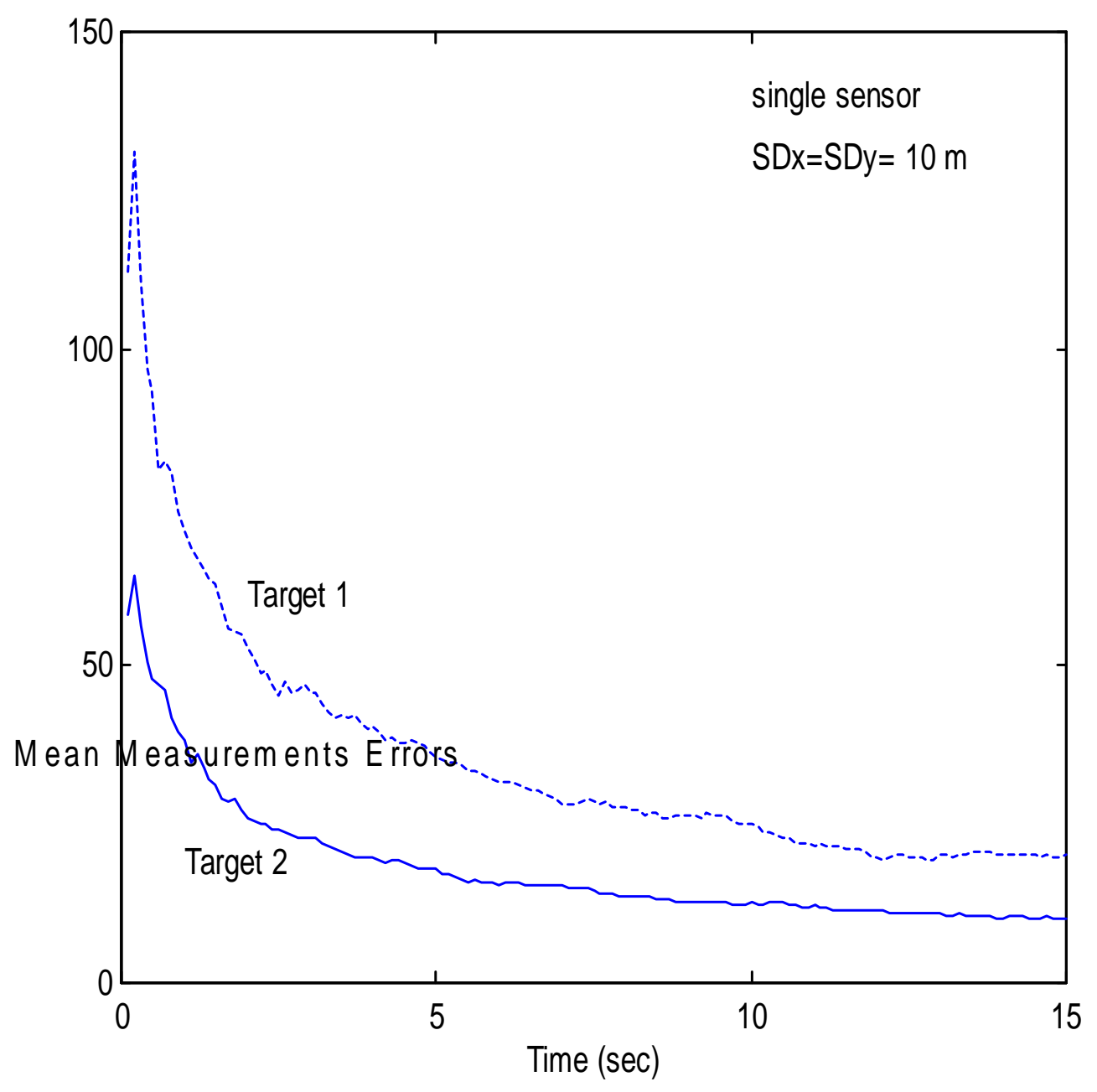

Fig. 7 Mean measurements errors in case of a single sensor 


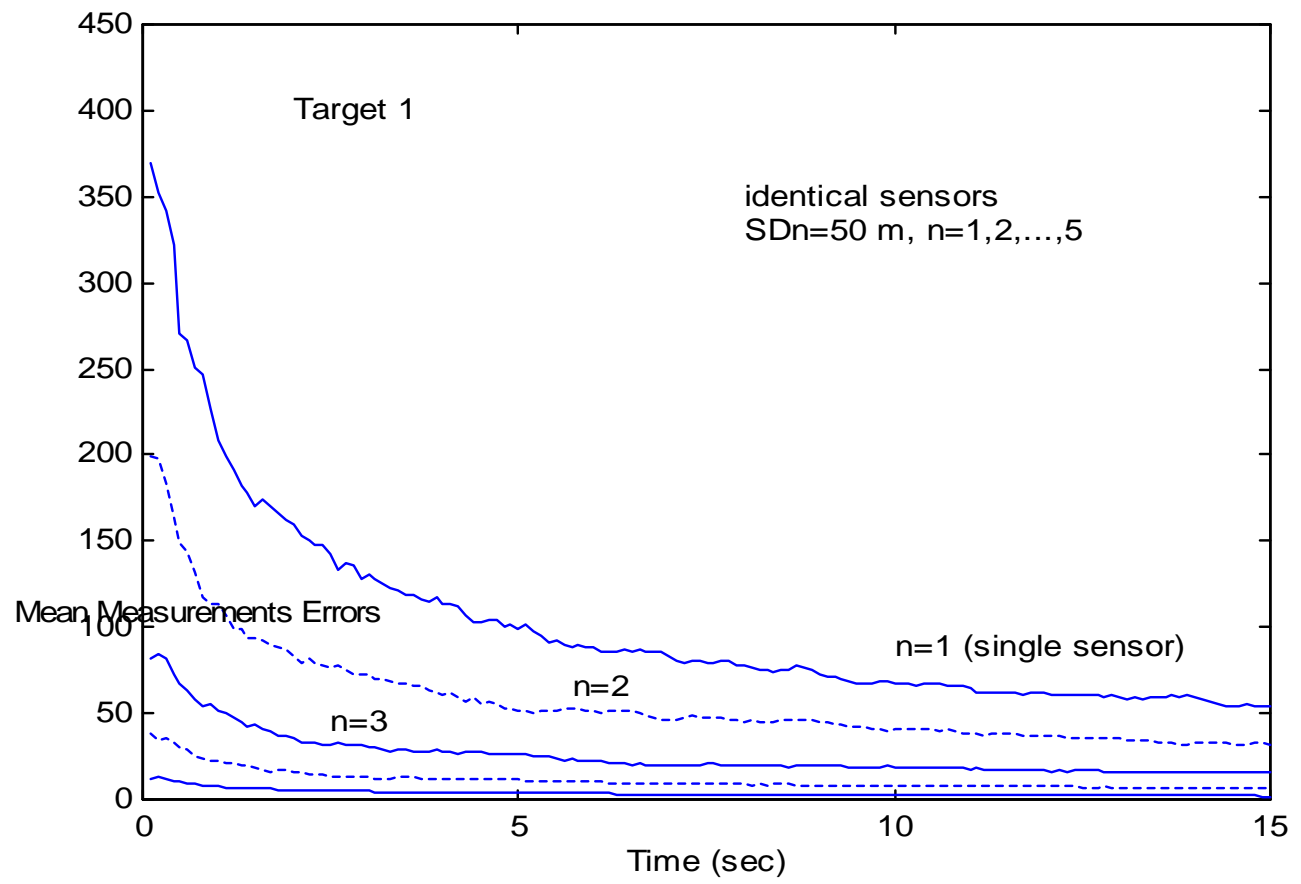

Fig.8 Mean measurements errors in case of multiple identical sensors

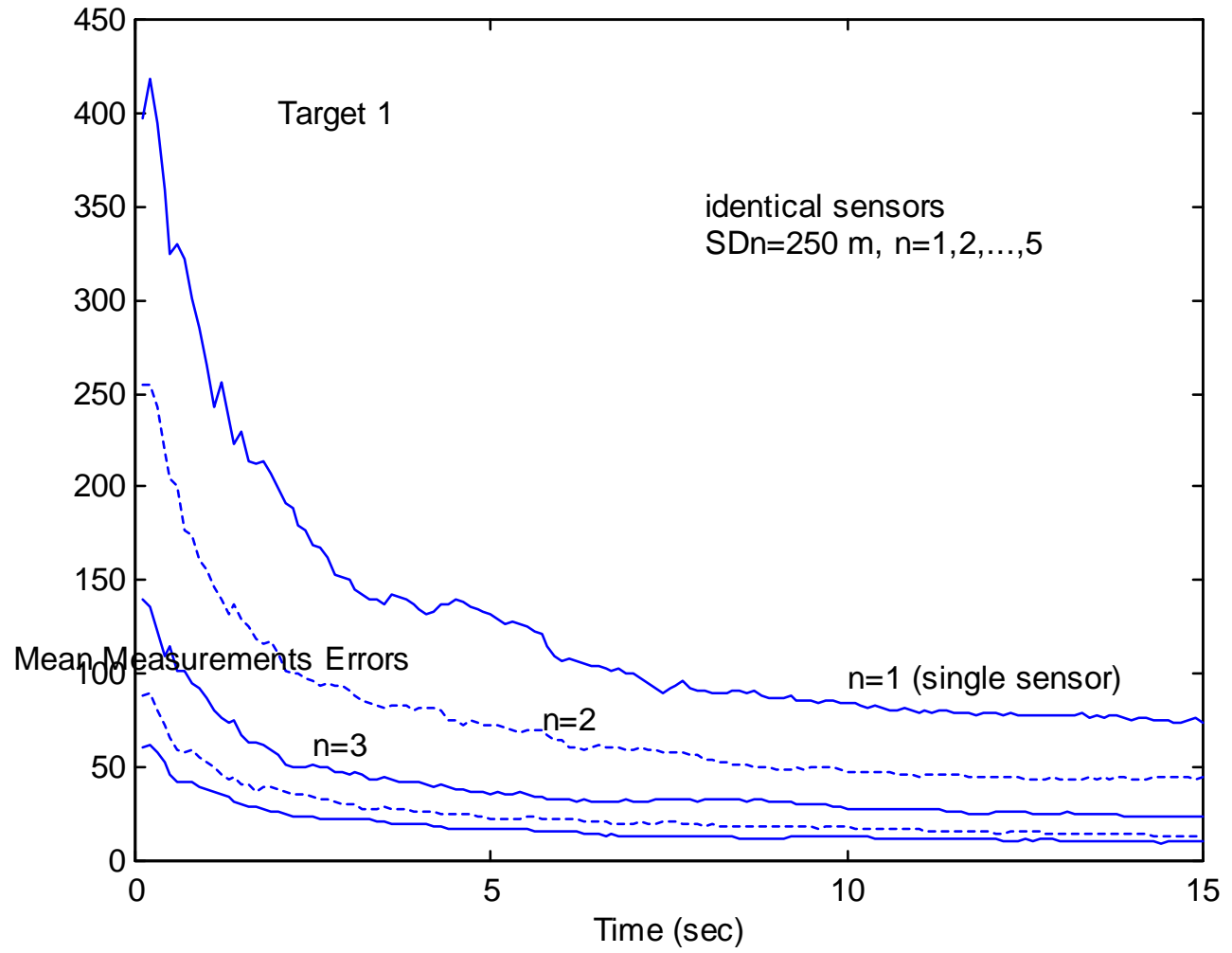

Fig. 9 Mean measurements errors in case of multiple identical sensors 


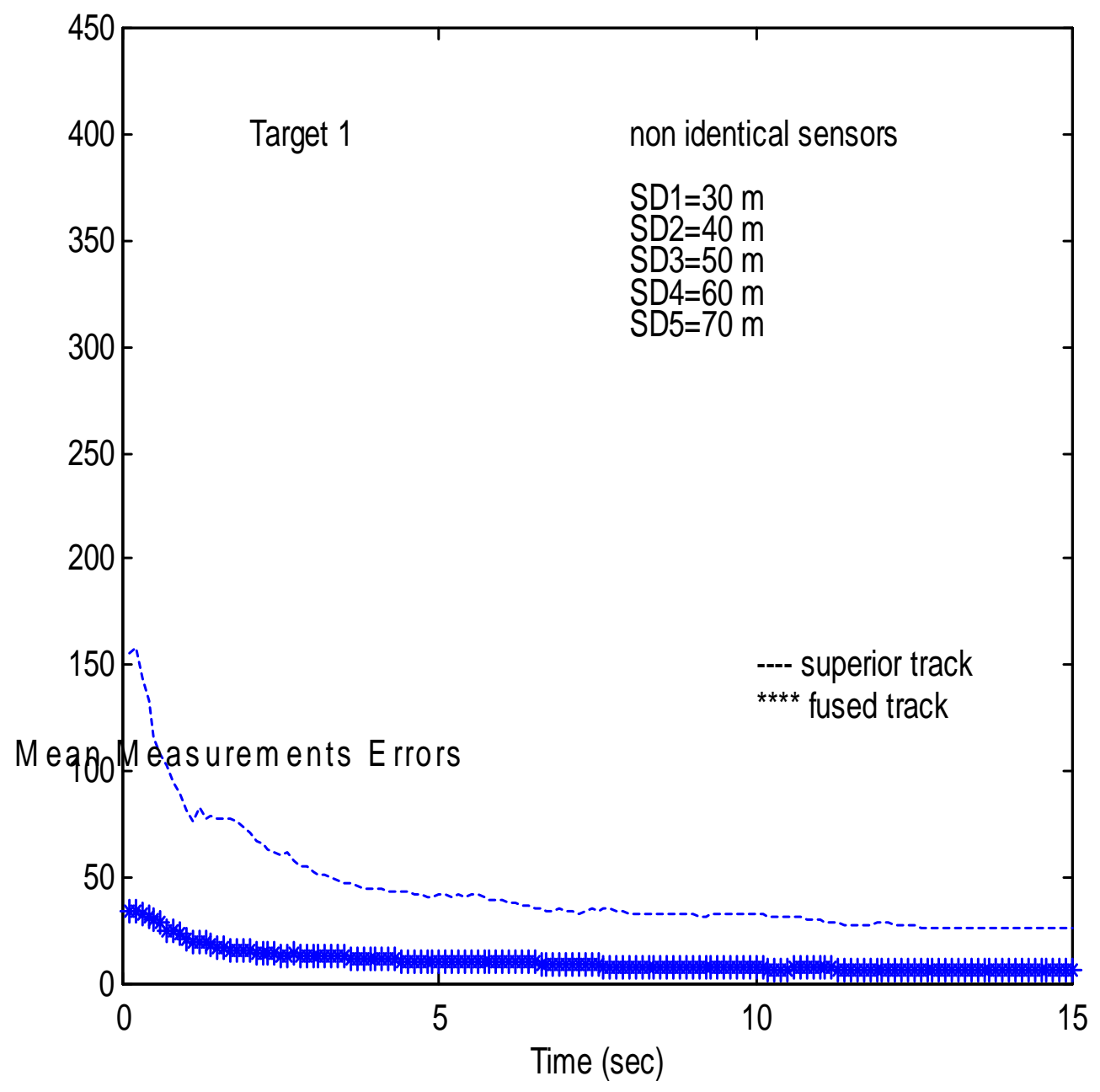

Fig. 10 Mean measurements errors in case of non identical sensors 


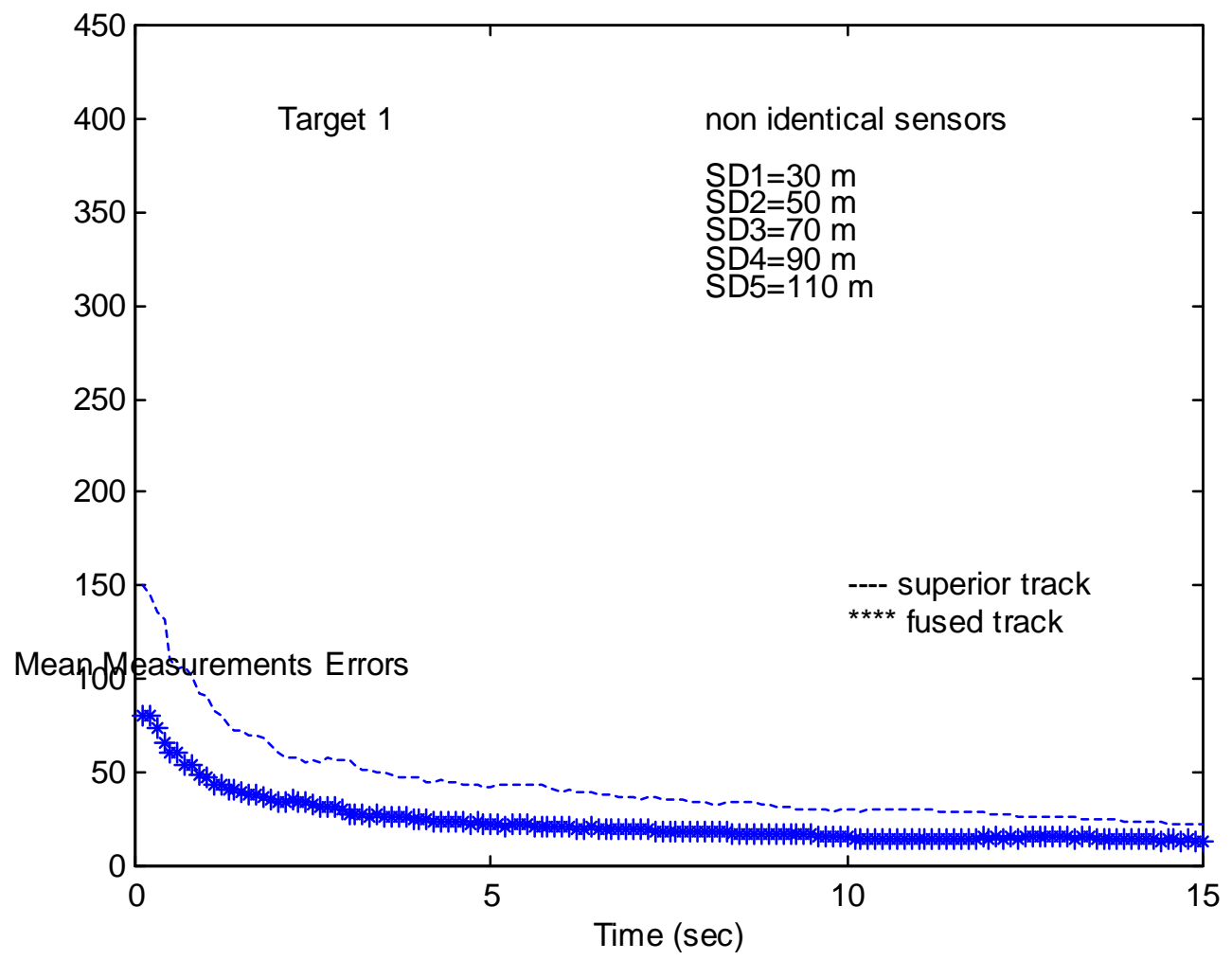

Fig. 11 Mean measurements errors in case of non identical sensors

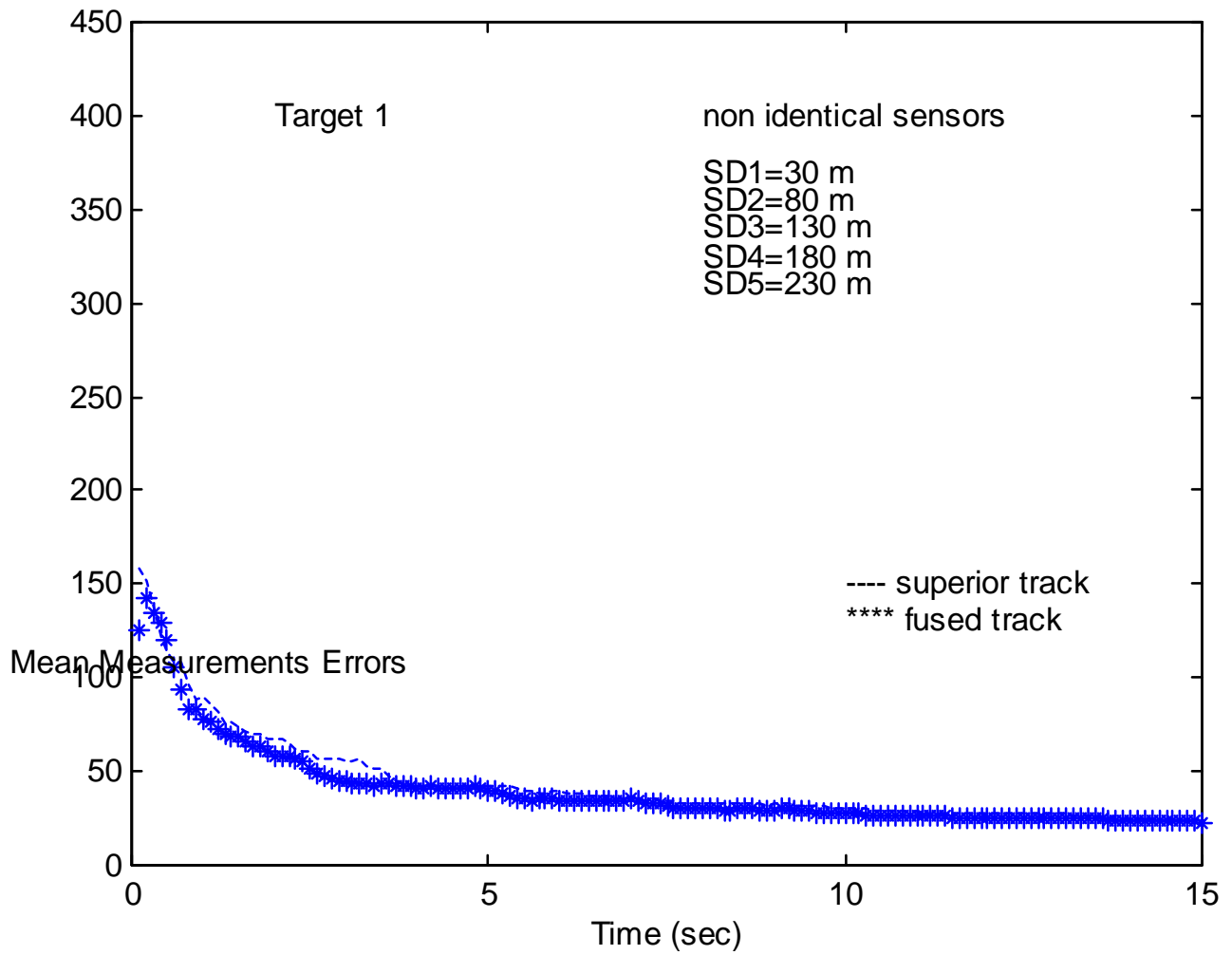

Fig. 12 Mean measurements errors in case of non identical sensors 


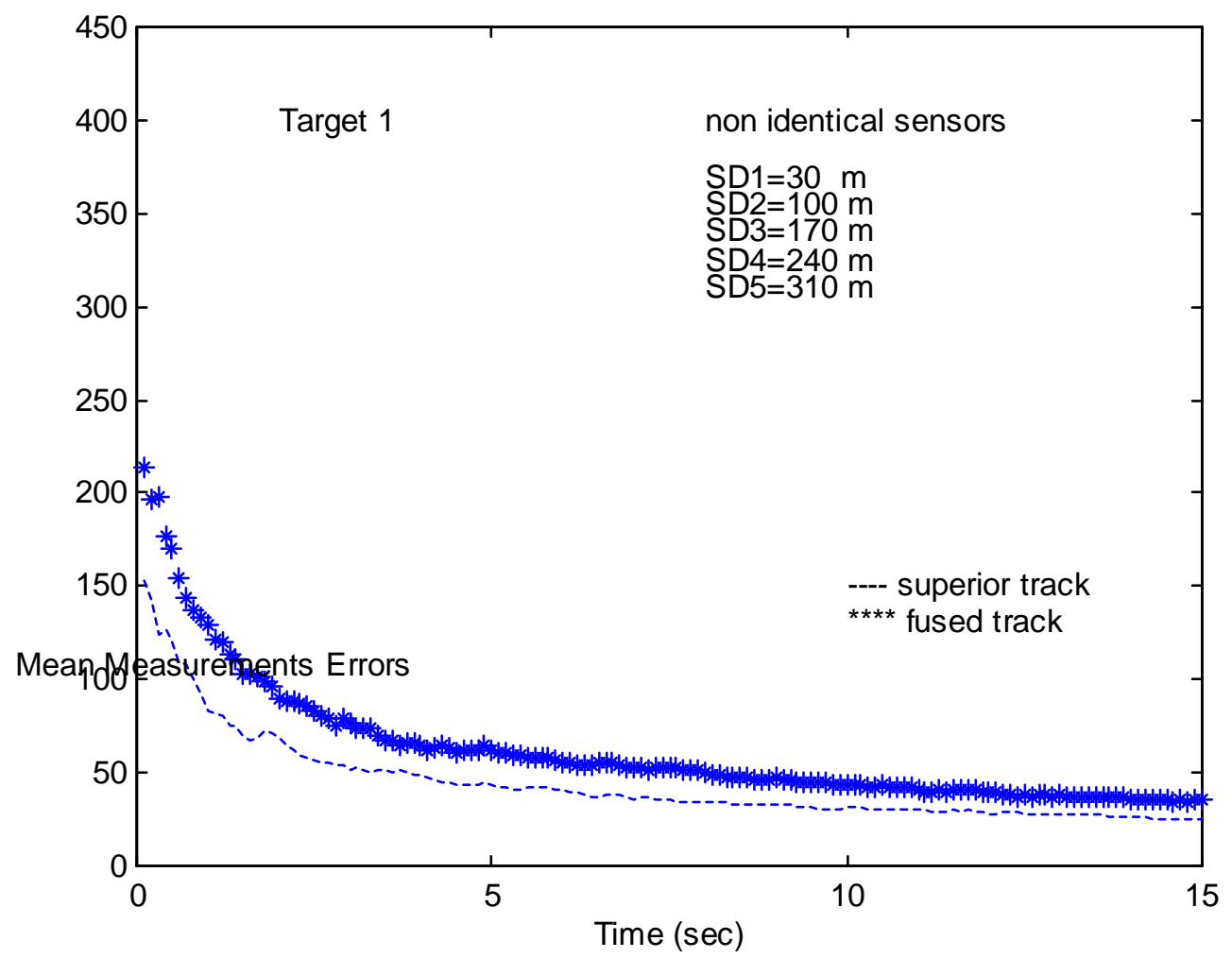

Fig. 13 Mean measurements errors in case of non identical sensors

\section{Conclusion:}

The data fusion model and its associated processing have been considered. The problem of position fusion, based on Bayesian minimum mean square criterion using measurements from dissimilar sensors, has been addressed. Performance evaluation of distributed trackers with multiple sensors in case of Gaussian distributed observations have been done using Monte Carlo simulations. Comparison of the performances of the fused track and the best estimate track has been also provided. The results show that under certain conditions the performance of the fused track may perform better than the performance of the better quality sensor estimate. It has been shown that the performance of the fused track is better than the performance of the superior track when the sensors have similar or comparable accuracies. It has been shown that the performance of the fused track may perform worse than the performance of the superior track when the sensor accuracies vary widely. In this case, adopting the superior track is recommended and tracks fusion is not recommended. The best performance of the fused estimate occurs when the distributed sensors are identical sensors. 


\section{REFERECNCES:}

[1] D. L. Hall, Mathematical Techniques in Multisensor Data Fusion, Norwood, MA, Artech House, 1992.

[2] J. Llinas, "Assessing the Performance of Multisensor Fusion Systems," Proceedings of SPIE, Vol. 1611, Sensor Fusion IV, 1991, pp. 2-27.

[3] E. Waltz and J. Llinas, Multisensor Data Fusion, Norwood, MA, Artech House, 1990.

[4] J. Llinas and D. Hall, "An Introduction to Multisensor Data Fusion," Proceedings of the 1998 IEEE International Symposium on Circuit and Systems, Volume 6, Monterey, CA, June 1998, pp. 537-540.

[5] S. S. Blackman, Multiple-Target Tracking With Radar Application, Norwood, MA, Artech House, 1986.

[6] Y. Bar-Shalom, Multitarget Multisensor Tracking: Applications and Advances, Volume I. Norwood, MA, Artech House, 1990.

[7] L. Martin, C. Chee-Yee, K. Ivan, A. Mark, V. Vincent and Thomopoulos, "Distributed Fusion Architectures and Algorithms for Target Tracking," Proceedings of the IEEE, Vol.85, NO.1, Jan. 1997.

[8] M. Krieg, et al., "Track Fusion in the Presence of an Interference," Forth International Symposium on Signal Processing and Its Applications, 1996, pp. 192-195.

[9] R. T. Antony, Principles of Data Fusion Automation, Norwood MA, Artech House, Boston, London, 1995.

[10] Y. Bar-Shalom and T. Fortman, Tracking and Data Association, Academic Press, 1988.

[11] T. Fortman, Y. Bar-Shalom, and M. Scheffe, "Multi-Target Tracking Using JPDA," Proceedings 1980 IEEE Conference on Decision and Control, December 1980, pp. 806-812.

[12] S. Deb, K. Pattipati, and Y. Bar-Shalom, "A Multisensor-Multitarget Data Association Algorithm for Heterogeneous Sensors," IEEE Transactions on Aerospace and Electronic Systems, Vol. 29, No. 2, April 1993.

[13] K. C. Chang and Y. BarShaloom, "FUSEDAT: A Software Package for Fusion and Data Association and Tracking With Multiple Sensors," Proceedings of SPIE, Vol. 2235, 1994, pp. 497-506.

[14] S. Xiaoquan, et al., "Data Association in Multitarget Tracking With Multisensor," Proceedings of the IEEE 1997 National Conference on Aerospace and Electronics, Vol. 2, 1997, pp. 884-889.

[15] Y. Bar-Shalom, "On the Track-to-Track Correlation Problem,"IEEE Transactions on Automatic Control, Vol. AC-26, No. 2, April 1981, pp. 571-572.

[16] R. K. Saha, "Track-to-Track Fusion With Dissimilar Sensors," IEEE Transactions on Aerospace and Electronic Systems , Vol. 32, No.3, July 1996, pp. 1021-1029.

[17] K. Chang, R. K. Saha, Y. Bar-Shalom, and M. Alford, 'Performance Evaluation of Multisensor Track-to-Track Fusion," Proceedings of the 1996 IEEE/SICE/RSJ 
International Conference on Multisensor Fusion and Integration for Intelligent Systems, 1996, pp. 627-632.

[18] R. K. Saha, "Effect of Cross-Correlation on Track-to-Track Fusion," Proceedings of SPIE , Vol. 2235, 1994, pp. 474-485.

[19] K. C. Chang, R. K. Saha and Y. Bar-Shalom, "On Optimal Track-to-Track Fusion," IEEE Transactions on Aerospace and Electronic Systems, Vol. 33, No. 4, October 1997, pp. 1271-1276.

[20] I. W. Dall and A. J. Shellshear, "Evaluation of Model Based Fusion Algorithm With Multi-Mode OTHR Data," IEEE Asilomar Conference on Signals, Systems, and Computers, 1993.

[21] S. Mori, W. H. Barker, C. Chong, and K. Chang, 'Track Association and Track Fusion With Nondeterministic target dynamics," IEEE Transactions on Aerospace and Electronic Systems, Vol. 38, No. 2, April 2002, pp 659-668.

[22] R. A. Singer and A. Kanyuck, "Correlation of Multi-Site Track Data," IEEE Transactions on Aerospace and Electronic Systems, AES-6, March 1970, pp. 180-187.

[23] R. A. Singer and A. J. Kanyuck, "Computer Control of Multiple-Site Track Correlation," Automatica, Vol. 7, 1971, pp. 455-463.

[24] R. A. Singer and K. Behnke, "Real-Time Tracking Filter Evaluation and Selection for Tactical Applications," IEEE Transactions on Aerospace and Electronic Systems, AES-7, No.1, Jan. 1971, pp. 100-110.

[25] D. Willner, B. Chang, and K. P. Dunn, 'Kalman Filter Algorithms for a MultiSensor System," Proceedings of IEEE Conference on Decision and Control, Dec. 1976, pp. 570-574.

[26] Y. Bar-Shalom and X. Rong Li, “Multitarget Multisensor Tracking: Principles and Techniques," IEEE Control Systems Magazine , Feb. 1996, pp. 93-96.

[27] A. M. Haimovich, J. Yosko, R. Greenberg, M. Parisi, and D. Becker, "Fusion of Sensors With Dissimilar Measurement/Tracking Accuracies,"IEEE Transactions on Aerospace and Electronic Systems, Vol. 29, No.1, Jan. 1993, pp. 245-250.

[28] F. R. Castella, "Theoretical Performance of a Multisensor Track-to-Track Correlation Techniques," IEE Proceedings on Radar, Sonar and Navigation, Vol. 142, No. 6, Dec. 1995, pp. 281-285.

[29] Y. Bar-Shalom and X. Rong Li, "Multitarget Multisensor Tracking: Principles and Techniques," IEEE AES Systems Magazine, Feb. 1996, pp. 41-44.

[30] Y. Bar-Shalom and X. Rong Li, Estimation and Tracking: Principles, Techniques, and Software, Artech House, Boston, London, 1993. 